\title{
A case report of a ectopic parathyroid adenoma and literature review
}

\section{Xue Liu ( $\nabla$ liuxue5436@163.com )}

the third people's Hospital of zhengzhou https://orcid.org/0000-0002-4459-4600

\section{lin Hui Wang}

the third people's hospital of zhengzhou

\section{Case report}

Keywords: Mediastinal mass, Compressed airway, Ectopic parathyroid adenoma

Posted Date: May 11th, 2021

DOl: https://doi.org/10.21203/rs.3.rs-448754/v1

License: (c) (i) This work is licensed under a Creative Commons Attribution 4.0 International License. Read Full License 


\section{Abstract \\ Background}

Giant ectopic mediastinal parathyroid adenoma with cystic degeneration and intratumoral hemorrhage is extremely rare, with low morbidity and a high misdiagnosis rate, and requires precise positioning and surgical resection. Case presentation: A 41-year-old woman presented with a sore throat for 3 days and hoarse voice for 1 day. The following chest computed tomography (CT) showed a large fusiform highdensity mass in the posterior tracheal space (maximum cross-section of $34 \times 25 \mathrm{~mm}$ ). ultrasonography of the neck showed a low-echo solid mass. This patient had undergone 2 puncture treatments and 1 surgical treatment from the discovery of the lesion to the diagnosis of the cause over the span of more than a month. Subsequently, the final pathology was definitely diagnosed as parathyroid adenoma with cystic lesions. Six months after the operation, the patient did not relapse.

\section{Conclusions}

This case reported a rare and huge ectopic mediastinal parathyroid adenoma that was misdiagnosed as mediastinal tumor hemorrhage at an early stage. For ectopic mediastinal parathyroid adenomas, surgery is the preferred treatment option. Warn us that we should expand the scope of disease thinking for mediastinal tumors.

\section{Introduction}

The parathyroid gland is the smallest endocrine organ, usually located on the back of the left and right lobes of the thyroid gland. There are 2 upper and lower pairs, with a total weight of approximately 120 mg. Parathyroid glands develop from the endoderm of the pharyngeal sac. The next pair of parathyroid glands, called ectopic parathyroid glands, comes from the third pharyngeal sac and can be connected to the thymus during their development and descend into the mediastinum ${ }^{[1]}$. Heterotopic anterior mediastinum is common and always confused with lymphoma, thymoma, dermoid cyst, or ectopic mediastinal thyroid. In the early stage, there are no symptoms or signs of hyperparathyroidism, so it is very easy to misdiagnose. This article describes an extremely rare case of a huge parathyroid gland that is located in the posterior mediastinum without any symptoms.

\section{Case Presentation}

The institutional review board of The Third People's Hospital of Zhengzhou approved this work. The patient provided informed consent for publication of the case. A 41-year-old woman presented with a sore throat for 3 days and hoarse voice for 1 day. One day before admission, a chest CT scan (Fig. 1A) showed a large fusiform high-density shadow in the posterior tracheal space (maximum cross-section of $34 \times 25 \mathrm{~mm}$, CT value $50 \mathrm{HU}$ ). The adjacent tracheal wall was compressed, the lumen was slightly 
flattened, and the boundary of the adjacent esophageal wall was partially unclear. Three days after admission, the patient gradually developed hoarseness, dry cough, wheezing, difficulty swallowing, and progressive swelling and tenderness on the right side of the face and neck. Chest 64-row enhanced CT (Fig. 1B) showed a soft tissue mass on the right side of the posterior superior mediastinum (uneven density, mild enhancement, CT value range of $-5 \mathrm{HU}-130 \mathrm{HU}$, and maximum cross-sectional area of $56 \times 36 \times 32 \mathrm{~mm}$ ), the pressure of the adjacent trachea was significantly higher than before, and the local wall of the middle and upper esophagus was thickened. Ultrasonography (US) of the neck (Fig. 1C) indicated a solid mass on the right side of the upper mediastinum near the upper sternal fossa (hypoechoic mass, maximum cross-sectional area of $52 \times 28 \times 25 \mathrm{~mm}$, clear border, irregular shape, deep part extending to the mediastinum, partly due to the obscurity of the sternum). The large blood vessels in the neck were immediately behind the upper edge, and the blood flow signal in the neck was unsatisfactory. The diagnosis was considered at this time, including bleeding from the right upper mediastinal mass, mediastinal metastasis of esophageal cancer and bleeding in metastatic foci, neurogenic tumor, or paratracheal cyst. Considering the rapid progression of the patient's condition, to alleviate the symptoms of local compression, the treatment plan was given, including (1) an emergency puncture to confirm the pathological diagnosis; (2) radiotherapy immediately after the puncture; and (3) methylprednisolone $40 \mathrm{mg} /$ day $\times 3$ days. Fiberoptic bronchoscopy was performed before the puncture, and the results (Fig. 1D) indicated a normal glottis and external pressure stenosis in the right posterior wall of the trachea. The upper end was approximately $1 \mathrm{~cm}$ from the glottis, and the lower end was approximately $2 \mathrm{~cm}$ from the carina. The upper section was narrowed by approximately $70 \%$, and the middle and lower sections were narrowed by approximately $40 \%$. The right posterior wall was slightly infiltrating, and mucosal congestion and edema were obvious. Based on the degree of airway compression seen in the patient under tracheoscopy, tracheal intubation was temporarily not performed. When the mediastinal mass was punctured under CT guidance, the tension in the mass was high, and 15 $\mathrm{ml}$ of dark red bloody non-coagulated liquid was withdrawn and submitted for pathological examination.

After the puncture, the patient felt chest tightness, hoarseness, and swelling of the right side of the face, so no radiotherapy was given. The post-pathological results revealed fibrous adipose tissue and a few glandular epithelial exfoliated cells, with no tumor cells detected. After further improvement of the relevant examinations, granulomatous disease, tuberculosis infection, thyroid disease, and cancer were excluded. At this time, the patient had been hospitalized for 13 days, and the cause of the mediastinal mass was still not detected. Reexamination of chest CT (Fig. 2A) showed that the soft tissue mass on the right side of the posterior superior mediastinum was smaller than that on the front. The pressure on the adjacent tracheal wall was significantly reduced. The thickening of the local wall of the middle and upper esophagus was slightly less than before. Reexamination of neck US (Fig. 2C) showed a cystic echogenic mass approximately $59 \times 26 \mathrm{~mm}$ deep on the right side of the superior sternal fossa, with poor internal translucency, no blood flow signal in it, and no local blood vessels. The margin was immediately adjacent to the inferior pole of the thyroid, and the lower margin was located at the upper edge of the aortic arch. Compared with the previous US, local compression was significantly alleviated. The lesion had a fluid echo, and a band-like separation echo was seen. In the thyroid consultation, the possibility of the patient 
having parathyroid cysts or parathyroid adenoma was considered. To further alleviate local compression and clarify the pathological diagnosis, under the guidance of ultrasound, a mass puncture was performed, and $20 \mathrm{ml}$ of dark red non-coagulating liquid was withdrawn. Then, US was immediately reexamined and showed that the mass shrunk significantly (Fig. 2D). Reexamination of chest CT (Fig. 2B) showed that the tracheal compression was almost completely relieved, and the mediastinal mass was significantly reduced. On the 14th day after admission, the pathological report of the second mediastinal puncture showed that the liquid sediment in the mediastinal cyst had a small number of glandular epithelial cell clusters, foam cells, lymphocytes and neutrophils in the background of the red blood cells prompted from cystic lesions of the parathyroid gland. Simultaneously, parathyroid hormone was $210 \mathrm{pg} / \mathrm{ml}$ (normal reference range: $11-81 \mathrm{pg} / \mathrm{ml}$ ). The results of ${ }^{99 \mathrm{~m}} \mathrm{Tc}-\mathrm{methoxy}$ isobutylisonitrile $\left({ }^{99 \mathrm{~m}} \mathrm{Tc}-\mathrm{MIBI}\right)$ examination of the parathyroid glands showed that there were abnormally increased areas of punctate radioactive distribution below the lower pole of the right lobe of the thyroid and the upper pole and lower pole of the left lobe, and the parathyroid imaging was suspiciously positive (possibly hyperparathyroidism). Then, the patient was transferred to the Surgery Department for surgical treatment. Postoperative pathology confirmed parathyroid adenoma with cystic changes in some areas (Fig. 2E and F). After 3 months of follow-up, the patient showed no abnormalities.

\section{Discussion And Conclusions}

Parathyroid adenoma (PA) is a benign parathyroid tumor. Hyperparathyroidism accounts for $85 \%-90 \%$ [2]. PA is mostly single, and rarely involves 2 glands. Approximately $90 \%$ of PA cases occur in the primary parathyroid gland. Cystic PA lesions account for approximately $4 \%-5 \%{ }^{[3]}$. Ectopic parathyroid adenoma $(E P A)^{[4,5]}$ accounts for approximately $5 \%-10 \%$ of primary PA, with an incidence of approximately $1.4 \%$. It is most common in the anterior mediastinum and is usually embedded in the thymus. Other cases can be found in the tracheoesophageal sulcus, posterior esophageal area, inferior thyroid pole, heart, etc. Based on the histopathology, PA can be divided into solid PA and cystic PA. Studies have suggested that functional parathyroid cysts may be the result of parathyroid adenoma cysts ${ }^{[6,7]}$. Therefore, cystic PAs are also called functional parathyroid cysts.

PA is divided into functional and nonfunctional parathyroid adenoma based on the presence or absence of parathyroid function. Functional PA pathological specimens show uneven thickening of the cyst wall, contents that are relatively thick, and a tan liquid-like "chocolate" substance. Laboratory tests show that high calcium, low phosphorus, and high parathyroid hormone levels can even cause hyperparathyroidism crisis. Functional PA can be diagnosed first in urology, orthopedics, and endocrinology because of urinary tract stones, bone pain, pathological fractures, and hypercalcemia accompanied by burnout, itching, and memory loss. If the PA is located in the tracheoesophageal sulcus, symptoms of dysphagia and airway compression can be seen. PA can cause intracapsular hemorrhage, local irritation and pain.

Nonfunctional PA pathological specimens show a thin cyst wall and contents that are clear and thin. Laboratory tests show no electrolyte abnormalities or parathyroid gland dysfunction. A retrospective study of 907 patients with $\mathrm{PA}^{[8]}$ showed that 870 patients had solid PA, and only 37 patients had cystic 
PA. The number of hypercalcemia crises in the cystic PA group was higher than that in the solid adenoma group. The preoperative serum total calcium and parathyroid hormone levels of cystic PA patients were significantly higher than those of solid PA patients. The maximum diameter of cystic PA was larger than that of solid adenoma. In terms of examination, the preoperative imaging methods of cystic PA, such as neck US and $99 \mathrm{mTc}-\mathrm{MIBI}$, have reduced accuracy ${ }^{[9]}$. Fine-needle puncture of the mass and determination of the parathyroid hormone content in the mass can help clarify ectopic parathyroid disease ${ }^{[10]}$.

Ectopic mediastinal parathyroid adenoma (MPA ${ }^{[11]}$ can cause false positives due to mediastinal metastatic lung cancer, thymoma, lymphoma, seminoma, etc. US cannot easily find these cases, which are difficult to diagnose. If the MPA is a nonfunctional adenoma or is located in the posterior mediastinum ${ }^{[12]}$ or if the mass is accompanied by bleeding, it is extremely easy to misdiagnose, further increasing the difficulty of diagnosis. However, the cause of bleeding in the lesion has not yet been clearly concluded and may be caused by adenoma hyperplasia and degeneration or insufficient blood supply and necrosis in the central area of the gland. However, hemorrhagic changes in parathyroid adenoma can cause functional changes in parathyroid glands ${ }^{[13]}$. In terms of treatment, current clinical studies have confirmed that for EPA, due to its special location, surgical resection is more complicated. Surgical treatment is required after precise positioning. The combined use of ${ }^{99 \mathrm{~m}} \mathrm{Tc}-\mathrm{MIBI}$ and anatomical imaging (CT/US/magnetic resonance imaging (MRI)) helps to accurately locate the ectopic parathyroid glands and improve the success rate of surgical treatment ${ }^{[4]}$. Video-assisted thoracoscopic surgery (VATS) is considered a safe and feasible option for the treatment of MPA ${ }^{[14,15]}$. Adenomas found above the upper mediastinal aortic arch can be successfully treated by a transcervical approach, while those found below the level of the aortic arch in the mediastinum or posterior mediastinum should be accessed through the thoracic cage ${ }^{[11]}$.

This patient had undergone 2 puncture treatments and 1 surgical treatment from the discovery of the lesion to the diagnosis of the cause over the span of more than a month. The reasons for the misdiagnosis of early disease mainly considered the following points. 1 . The patient had no typical symptoms of hyperparathyroidism and no abnormalities in the anatomical imaging examination at the early stage of the disease and only showed local compression, which was a huge ectopic mediastinal parathyroid adenoma with cystic changes. This case was extremely rare and easily misdiagnosed. 2 . The patient's mediastinal mass was located in the posterior mediastinum, which was more common with neurogenic tumors. 3. Early chest-enhanced CT showed thickening of the esophageal wall with a mediastinal mass, which did not exclude the possibility of esophageal cancer metastasis. 4 . In the early stage of symptoms, bleeding occurred in the mass, which resulted in an increased CT value. The first US showed a solid mass. The disease progressed faster, and the symptoms became significantly worse. There was a possibility of life crisis at any time, so the possibility of tumor necrosis was not excluded, which further increased the misdiagnosis rate of the disease, which led to the delay of the diagnosis.

\section{Declarations}


Acknowledgements:

Not applicable.

\section{Funding:}

There was no financial support.

\section{Availability of data and materials:}

The work is original research that has not been published previously and not under consideration for publication elsewhere, in whole or in part. Data is shared during the current study.

\section{Author contributions:}

Xue Liu collected all the data and authored the manuscript. Huilin Wang was responsible for the analysis of follow-up data. All authors had read and approved the final version of the manuscript.

\section{Ethics approval and consent to participate:}

The study was approved by the Ethical Committee of The Third People's Hospital of Zhengzhou, Henan and was conducted according to the principles of the Declaration of Helsinki.

\section{Consent for publication:}

The patient granted written informed consent for publication of this manuscript and the accompanying images.

\section{Competing interests:}

This material has not been published and is not under consideration elsewhere. There is no financial disclosure from each author. The authors declare that they have no competing interests.

\section{References}

1. Salido S, Gómez-Ramírez J, Bravo JM, et al. Parathyroid adenoma in third pharyngeal pouch cyst as a rare case of primary hyperparathyroidism. Annals of the Royal College of Surgeons of England. Oct 2014;96(7):e8-10. doi: 10.1308/003588414X13946184900804.

2. Baloch ZW, LiVolsi VA. Pathology of the parathyroid glands in hyperparathyroidism. Seminars in diagnostic pathology. Aug 2013;30(3):165-177. doi: 10.1053/j.semdp.2013.06.003.

3. Rickes S, Schmidt B, Gerl H, Lochs H. Unusual localization in primary hyperparathyroidism. Medizinische Klinik (Munich, Germany : 1983). Aug 15 2000;95(8):461-465.doi:

10.1007/s000630050009. 
4. Noussios G, Anagnostis P, Natsis K. Ectopic Parathyroid Glands and their Anatomical, Clinical and Surgical Implications. Exp Clin Endocrinol Diabetes. 2012;120(10):604-610. doi: 10.1055/s-00321327628.

5. Zerizer I, Parsaï A, Win Z, Al-Nahhas A. Anatomical and functional localization of ectopic parathyroid adenomas: 6-year institutional experience. Nuclear medicine communications. Jun 2011;32(6):496502. doi: 10.1097/MNM.0b013e32834557a3.

6. Mccoy KL, Yim JH, Zuckerbraun BS, Ogilvie JB, Peel RL, Carty SE. Cystic parathyroid lesions: functional and nonfunctional parathyroid cysts. Arch Surg. 2009;144(1):52-56. doi: 10.1001/archsurg.2008.531.

7. Yalcin Y, Mete T, Aktimur R, et al. A Case of Primary Hyperparathyroidism due to Intrathyroidal Parathyroid Cyst. Case Rep Endocrinol. 2014;2014:213283. doi: 10.1155/2014/213283.

8. Hu Y, Cui M, Yu X, et al. The Clinical Features of Cystic Parathyroid Adenoma in Chinese Population: A Single-Center Experience. International Journal of Endocrinology. 2018;2018: 3745239. doi: $10.1155 / 2018 / 3745239$.

9. Gotthardt M, Lohmann B, Behr TM, et al. Clinical Value of Parathyroid Scintigraphy with Technetium99m Methoxyisobutylisonitrile: Discrepancies in Clinical Data and a Systematic Metaanalysis of the Literature. World Journal of Surgery. 2004;28(1):100-107. doi: 10.1007/s00268-003-6991-y.

10. Triggiani V, Resta F, Giagulli VA, et al. Parathyroid hormone determination in ultrasound-guided fine needle aspirates allows the differentiation between thyroid and parathyroid lesions: our experience and review of the literature. Endocrine, metabolic \& immune disorders drug targets. Dec 2013;13(4):351-358. doi: 10.2174/1871530313666140108125645.

11. Hu J, Ngiam KY, Parameswaran R. Mediastinal parathyroid adenomas and their surgical implications. Annals of the Royal College of Surgeons of England. May 2015;97(4):259-261. doi: 10.1308/003588415X14181254789088.

12. Güneş SG, İbrahimov F, Uğurum Yücemen A, Yenigün BM, Enön S. Atypically located cystic parathyroid adenoma. Turk gogus kalp damar cerrahisi dergisi. Jan 2020;28(1):217-218. doi: 10.5606/tgkdc.dergisi.2020.17627.

13. Uehara A, Suzuki T, Yamamoto Y, et al. A Functional Parathyroid Cyst from the Hemorrhagic Degeneration of a Parathyroid Adenoma. Internal medicine (Tokyo, Japan). Feb 1 2020;59(3):389394. doi: 10.2169/internalmedicine.3319-19.

14. Alesina PF, Moka D, Mahlstedt J, Walz MK. Thoracoscopic removal of mediastinal hyperfunctioning parathyroid glands: personal experience and review of the literature. World J Surg. Feb 2008;32(2):224-231. doi: 10.1007/s00268-007-9303-0.

15. Kim YS, Kim J, Shin S. Thoracoscopic removal of ectopic mediastinal parathyroid adenoma. The Korean journal of thoracic and cardiovascular surgery. Jun 2014;47(3):317-319. doi: 10.5090/kjtcs.2014.47.3.317.

\section{Abbreviations}


EPA Ectopic parathyroid adenoma

MPA Ectopic mediastinal parathyroid adenoma

MRI magnetic resonance imaging

PA Parathyroid adenoma

99mTc-MIBI 99m Tc-methoxyisobutylisonitrile

US ultrasonography

VATS Video-assisted thoracoscopic surgery.

\section{Figures}
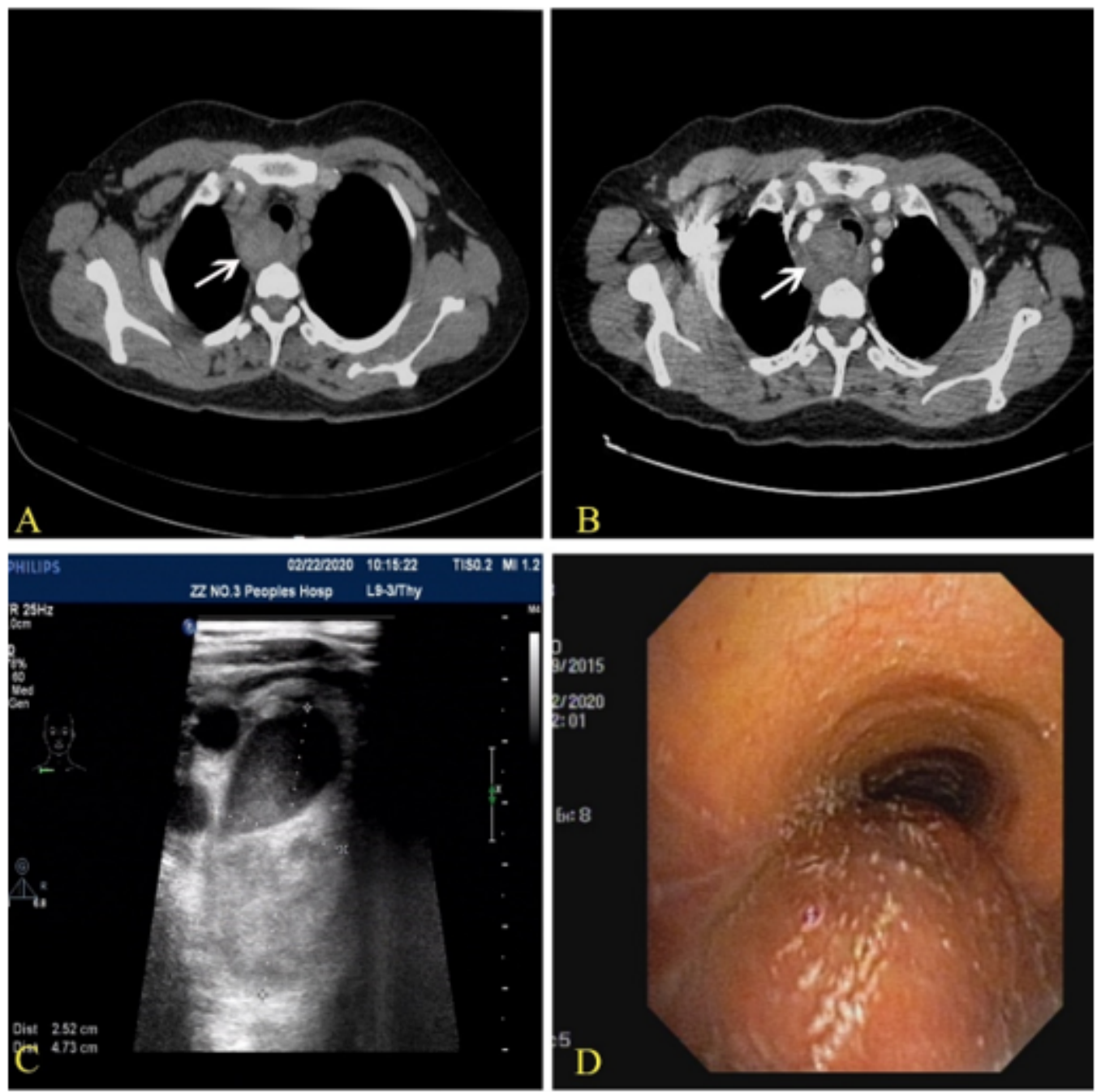

Figure 1 
(A) A 16-row CT scan of the chest revealed a fusiform high-density shadow (white arrow) in the posterior tracheal space, which was slightly compressed near the trachea, with a maximum cross-sectional area of $34 \times 25 \mathrm{~mm}$. (B) Chest 64-row enhanced CT revealed uneven density of the mass (white arrow, maximum cross-sectional area of $56 \times 36 \times 32 \mathrm{~mm}$ ), which was slightly enhanced. The pressure on the adjacent trachea was significantly higher than before. (C) Ultrasound showed a solid hypoechoic mass. The boundary was clear, the shape was irregular, and the deep part extended to the mediastinum. (D) Fibrous bronchoscopy showed external pressure stenosis of the right posterior wall of the trachea. CT =computed tomography, US = ultrasonography.
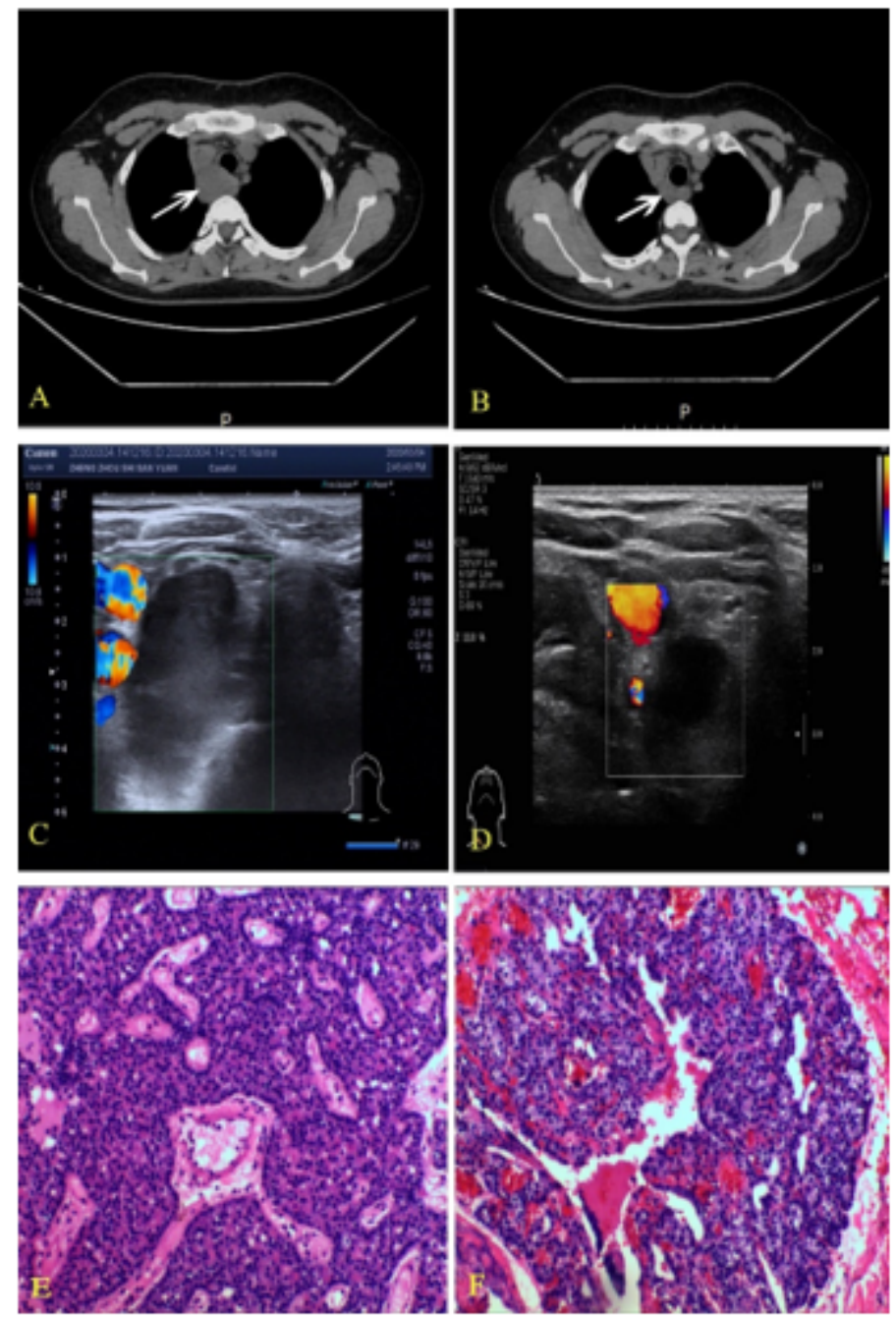

Figure 2

Ultrasound-guided anatomical imaging examination and pathological results before and after the puncture of mediastinal masses. (A) Chest CT before the puncture showed that the mass (white arrow) in the posterior tracheal space was smaller than before, and the adjacent trachea was less compressed than before. (B) After the puncture, chest CT showed that the mass (white arrow) in the space behind the trachea was significantly smaller than before, and the adjacent trachea was relieved of pressure. (C) Before the puncture, US showed a cystic echogenic mass with a size of approximately 59x26 mm. The upper edge was next to the inferior thyroid pole, and the lower edge was located at the upper edge of the 
aortic arch. The mass had a fluid echo, and a band-like separation echo was observed. (D) After the puncture, US showed that the cystic echogenic group was significantly reduced. (E, F) Pathological tissue sections showed parathyroid adenoma with partial cystic lesions (H\&E; original magnification, $\mathrm{x} 100$ ). $\mathrm{H} \& \mathrm{E}=$ hematoxylin and eosin, CT =computed tomography, US =ultrasonography.

\section{Supplementary Files}

This is a list of supplementary files associated with this preprint. Click to download.

- coverletter.docx 